\title{
Laparoscopic removal of the cervical stump for a cervical solid mass in a patient with previous supracervical hysterectomy
}

\author{
(D) Burak Sezgin ${ }^{1}$, (D) Aysun Camuzcuoğlu², (D) Hakan Camuzcuoğlu² \\ 1Department of Obstetrics and Gynecology, Muğla Sitkı Koçman University Faculty of Medicine, Muğla, Turkey \\ ${ }^{2}$ Clinic of Obstetrics and Gynecology, Private Adatıp Sakarya Hospital, Sakarya, Turkey
}

\section{Abstract}

The aim of this video article is to demonstrate a surgical tutorial in which a cervical mass was resected with laparoscopic removal of the cervical stump after supracervical hysterectomy ( $\mathrm{SCH}$ ). First, the dense adhesions due to previous operation were dissected. Then the dissection was continued carefully in order to identify bilateral ureters and iliac arteries. A cervical mass of nearly $4 \mathrm{~cm}$ was identified at the posterosuperior aspect. The mass was separated from surrounding tissue and removal of the cervical stump was performed. This is an exciting case, illustrating that minimally invasive procedures, such as laparoscopic removal of the cervical stump after SCH, can be used in the management of benign cervical tumor. The main feature of this operation was to check the relationship of tumor with adjacent structures, including ureters, bowel and vascular structures at every step to prevent unwanted injuries.

Keywords: Cervical mass, laparoscopy, cervical stump

Received: 17 August, 2019 Accepted: 02 December, 2019

\section{Introduction}

Laparoscopic removal of the cervical stump was first reported by Nezhat et al. (1). It can be considered a definitive therapy for patients with recurrent symptoms after supracervical hysterectomy ( $\mathrm{SCH}$ ) (2). There are some significant risk factors, including younger age, pelvic pain and endometriosis, for removal of the cervical stump in patients who have previously undergone $\mathrm{SCH}$ (3). It can be performed vaginally, abdominally or laparoscopically and either with or without robotic assistance. If a laparoscopic approach is chosen, advanced experience on laparoscopy is crucial (1). Here, we present a surgical tutorial in which a cervical mass was resected, with laparoscopic removal of the cervical stump (Video 1).

A 50-year-old G2P1 woman was referred to our hospital with the complaint of pelvic pain for three months. She had a history of $\mathrm{SCH}$ for abnormal uterine bleeding due to myoma uteri 10 years previously. Bimanual pelvic examination revealed tenderness and vaginal speculum examination was normal. A solid mass of nearly $4 \times 4 \mathrm{~cm}$ was identified at the cervical stump on transvaginal ultrasound. Laboratory tests for tumor biomarkers were negative while magnetic resonance imaging revealed a $4 \mathrm{~cm}$ in diameter solid mass localized at the cervical region. The patient underwent gastroscopy and colonoscopy because of malignancy suspicion but no abnormality was detected. Endocervical biopsy and Pap smear results revealed no abnormality. After fully informing the patient, consent was given for the use of images and videos of the procedure.

After the patient preparation, a uterine manipulator was inserted vaginally with a colpotomizer. A $10 \mathrm{~mm}$ umbilical port and three $5 \mathrm{~mm}$ abdominal ports were placed and the pelvis was explored. There were extremely dense adhesions between bowel, omentum and the anterior abdominal wall. First, the dense adhesions, due to the previous operation, were dissected. Then the dissection was carefully continued in order to identify bilateral ureters and iliac arteries (Figure 1). The 
previously identified $4 \mathrm{~cm}$ cervical mass at the posterosuperior aspect was identified (Figure 2). The mass was separated from surrounding tissue and a laparoscopic removal of the cervical stump was performed. After this procedure, the bladder was investigated with methilene blue for any signs of injury. The operation time was 185 minutes. There were no complications. The postoperative course was uneventful and the patient was discharged on the third postoperative day. Histopathological examination confirmed a benign, $4 \mathrm{~cm}$ leiomyoma.

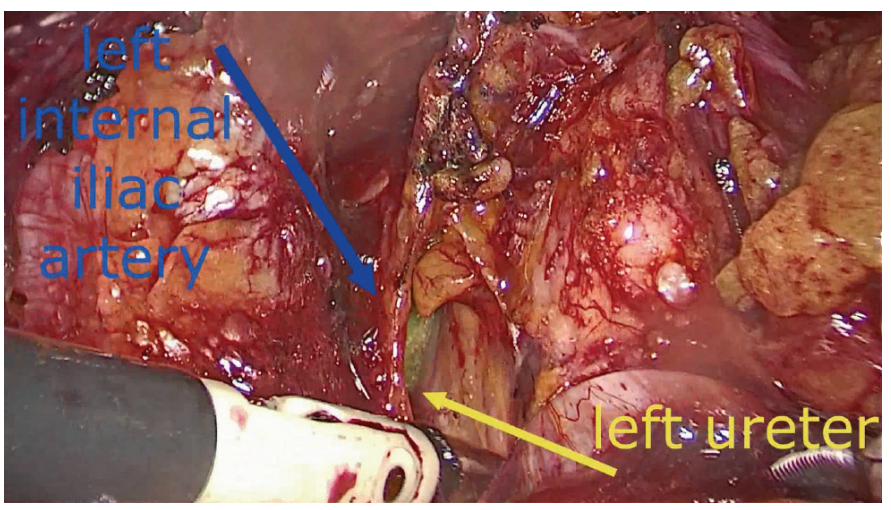

Figure 1. Appearance of left ureter and left internal iliac artery

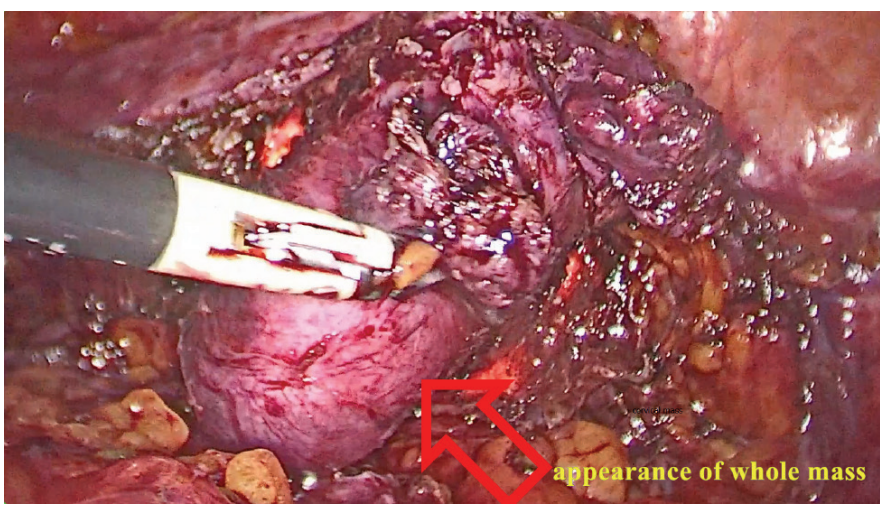

Figure 2. Appearance of the whole mass
This is an exciting case illustrating that minimally invasive procedures, such as laparoscopic removal of the cervical stump after $\mathrm{SCH}$, can be used in the management of benign cervical tumor. The main feature of this operation was to check the relationship of tumor with adjacent structures, including ureters, bowel and vascular structures at every step to prevent unwanted injuries.

\section{Video 1.}

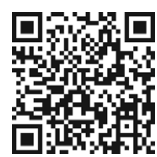

https://www.doi.org/10.4274/jtgga.galenos.2019.2019.0143.video1

Conflict of Interest: No conflict of interest was declared by the authors.

Financial Disclosure: The authors declared that this study received no financial support.

\section{References}

1. Nezhat $\mathrm{CH}$, Nezhat F, Roemisch M, Seidman DS, Nezhat C. Laparoscopic trachelectomy for persistent pelvic pain and endometriosis after supracervical hysterectomy. Fertil Steril 1996; 66: 925-8.

2. Tsafrir Z, Aoun J, Hanna R, Papalekas E, Schiff L, Theoharis E, et al. Robotic trachelectomy after supracervical hysterectomy for benign gynecologic disease. JSLS 2016; 20: e2016.00027.

3. Tsafrir Z, Aoun J, Papalekas E, Schiff LD, Theoharis E, Hanna R, et al. Risk factors for Trachelectomy Following Supracervical Hysterectomy. J Minim Invasive Gynecol 2015; 22: S82-3. 